\title{
CAEP/ACMU 2015 Research Awards
}

First place, Plenary Presentation, Grant Innes Research Paper and Presentation Award

Debra Eagles

PL01 Timed up and go in elderly emergency department patients following minor trauma

\section{Second place, Plenary Presentation}

Paul Atkinson

PL02 Sonography in cardiac arrest: Real-time Assessment and Evaluation with Sonography - Outcomes Network (REASON)

\section{Third place, Plenary Presentation}

Aikta Verma

PL03 Push-alert notifications of troponin results to physician smartphones: impact on emergency department patient flow

Fourth place, Plenary Presentation, Top Resident Abstract Award

Robert Ohle

PL04 Factors associated with choosing the emergency department as a primary access point to health care in a population without a primary care physician: a Canadian cross sectional study

\section{Top New Investigator Award}

Venkatesh Thiruganasambandamoorthy

OP02 Prognostic value of cardiac biomarkers in the risk-stratification of ED syncope - a Systematic Review

\section{Top Pediatric Abstract Award \\ Graham Thompson}

OP29 Magnetic resonance imaging provides useful diagnostic information following equivocal ultrasound in children with suspected appendicitis

\section{Top Medical Student Project Award}

Colin Rouse

LO10 A traumatic tale of two cities: Does EMS level of care and transportation model affect survival in trauma patients transported to level 1 trauma centres?

\section{Top Education Innovation Abstract Award}

Lynsey Martin

OP18 An emergency medicine residency program as emergency medical services medical advisor: an evaluation of curriculum effectiveness

\section{CAEP Resident Research Abstract Awards}

Christopher Lee

LO32 First-responder accuracy using SALT during mass-casualty incident simulation

Shahbaz Syed

OP22 Prospective validation of a clinical decision rule to identify which chest pain patients can safely be removed from cardiac monitoring in the emergency department

Simon Jones

LO19 Variability of CTAS scoring in two tertiary care centres in Calgary

Lauren Shephard

LO12 Morbidity and mortality associated with prehospital "lift assist" calls.

Jessica Moe

OP10 Effectiveness of interventions to decrease emergency department visits by adult frequent users: a systematic review

Neil Dattani

LO23 Frailty and the use of health services by older patients following a minor injury 
Tristan Snider

OP37 Comfort with geriatric emergency medicine competencies: a survey of Canadian emergency medicine residents

\section{CAEP Research Grant Recipients}

Clare Atzema

The safety of emergency department cardioversion: Results from the Atrial Fibrillation in the Emergency Room (AFTER) study

Kaitlin Graham

Incidence of clinically relevant medication discrepancies after implementation of an electronic medication reconciliation process
Michael Ho

The utility of ECG characteristics as prognostic markers in pulseless electrical activity arrests: a retrospective observational cohort study

Robert Ohle

A retrospective case control study to identify high risk clinical factors for aortic dissection

Catherine Patocka

The impact of spaced instruction on Emergency Medical Services (EMS) provider long-term retention of pediatric resuscitation performance: a randomized controlled trial (RCT) 